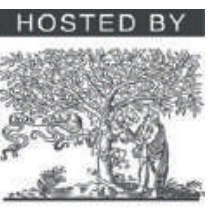

\title{
Hybrid firefly and Particle Swarm Optimization algorithm for the detection of Bundle Branch Block
}

\author{
Padmavathi Kora ${ }^{\text {a,* }}$, K. Sri Rama Krishna ${ }^{\text {b }}$ \\ a Gokaraju Rangaraju Institute of Engineering and Technology, Hyderabad 500090, India \\ ${ }^{\mathrm{b}}$ VR Siddardha Engineering College, Vijayawada 520007, India
}

\section{A R T I C L E I N F O}

\section{Article history:}

Received 11 September 2015

Accepted 6 December 2015

Available online 17 December 2015

\section{Keywords:}

Bundle Branch Block

FFA

PSO

FFPSO

LM NN classifier

\begin{abstract}
A B S T R A C T
Abnormal Cardiac beat identification is a key process in the detection of heart ailments. This work proposes a technique for the detection of Bundle Branch Block (BBB) using hybrid Firefly and Particle Swarm Optimization (FFPSO) technique in combination with Levenberg Marquardt Neural Network (LMNN) classifier. BBB is developed when there is a block along the electrical impulses travel to make heart to beat. ECG feature extraction is a key process in detecting heart ailments. Our present study comes up with a hybrid method combining the two meta-heuristic optimization methods, Firefly algorithm (FFA) and Particle Swarm Optimization (PSO), for feature optimization of ECG (BBB and normal) patterns. One of the major controlling forces is the light intensity attraction of FFA algorithm that models the optimum solution. The light intensity attraction process of the FFA algorithm depends on random directions for search, this may delay in achieving the global optimization solution. The hybrid technique FFPSO, integrates the concepts from FF algorithm and PSO and creates new individuals. In the FFPSO method the local search is performed through the modified light intensity attraction step with PSO operator. The FFPSO features are compared with the classical FF, PSO features. The FFPSO feature values are given as the input to the Levenberg Marquardt Neural Network (LM NN) classifier. It has been observed that the performance of the classifier is improved with the help of the optimized features.

(c) 2015 The Society of Cardiovascular Academy. Production and hosting by Elsevier B.V. All rights reserved. This is an open access article under the CC BY-NC-ND license (http://creativecommons.org/licenses/by-nc-nd/4.0/).
\end{abstract}

\section{Introduction}

Electro-cardiogram is used to access the electrical activity of a human heart. The diagnosis of the heart ailments by the doctors is done by following a standard changes. In this project our aim is to automate the above procedure so that it leads to correct diagnosis. Early diagnosis and treatment is of great importance because immediate treatment can save the life of the patient. BBB is a type of heart block in which disruption to the flow of impulses through the right or left bundle of His, delays activations of the appropriate ventricle that widens QRS complex and makes changes in QRS morphology. The changes in the morphology can be observed through the changes in the ECG. Good performance depends on the accurate detection of ECG features. ECG changes in Left Bundle Branch Block (LBBB) are:

- Increased QRS complex duration ( $\geq 0.12 \mathrm{~s})$

- Increased Q wave amplitude

- Abnormal T wave

ECG changes in Right Bundle Branch Block (RBBB) are:

\footnotetext{
* Corresponding author.

Peer review under responsibility of The Society of Cardiovascular Academy.
}

- Increased QRS complex duration $(\geq 0.12 \mathrm{~s})$

- RSR' format

- T wave inversion

Detection of BBB using ECG involves three main steps: preprocessing, feature extraction and classification. The first step in preprocessing mainly concentrates in removing the noise from the signal using filters. The next step in the preprocessing is the ' $R$ ' peak detection then these ' $R$ ' peaks are used to segment the ECG file into beats. The samples that are extracted from each beat contain non-uniform samples. The non-uniform samples in each beat are converted into uniform samples of size 200 by using a technique called resampling. The resampled ECG beat.

In the feature extraction procedure, a fraction of signal around the $\mathrm{R}$ peak is extracted as the time-domain features since the R peak of ECG signals is an important index for cardiac diseases. To ensure the important characteristic points of ECG like P, Q, R, S and T are included, a total of 200 sampling points before and after the $\mathrm{R}$ peak are collected as one ECG beat sample.

$\mathrm{P}, \mathrm{Q}, \mathrm{R}, \mathrm{S}$ and $\mathrm{T}$ waves provides information regarding amplitudes and relative time intervals of ECG. These changes in the ECG are called morphological transitions. The morphological changes (P, QRS complex, $\mathrm{T}, \mathrm{U}$ waves) of ECG are due to the abnormalities in the heart. BBB is one 
such morphological abnormality seen in the heart diseases. In the previous studies morphological features are extracted for clinical observation of heart diseases. The feature extraction using traditional techniques generally yield a large number of features, and many of these might be insignificant. Therefore, the common practice is to extract key features useful in the classification.

This paper presents meta-heuristic FFPSO, is used as a feature extraction method instead of using traditional feature extraction/optimization techniques. A large number of meta-heuristic techniques have been designed to solve feature optimization problem. Some of the methods among all these are Genetic Algorithm (GA), ${ }^{7}$ Particle Swarm Optimization (PSO), ${ }^{6}$ Bacterial Foraging Optimization(BFO), ${ }^{4,5,3}$ Firefly Algorithm $(\text { FFA })^{13}$ etc.

Meta-heuristic algorithms are proven to outperform the gradient based algorithms for real world optimization problems. Firefly algorithm ${ }^{1}$ is one such newly designed algorithm mimicking flashing mechanism of fireflies. A detailed explanation and formulation of the firefly algorithm is given in Section 4 .

Traditional Firefly Algorithm (FFA) ${ }^{2}$ has one disadvantage of getting trapped into the local optimum. Sometimes it is unable to come out of that state. The parameters in the firefly algorithm are fixed and do not have any mechanism to remember the previous best situation of each firefly and this makes them move regardless of its previous better solution.

In this paper, a novel hybrid optimization method concurrently combines the FFA with the PSO. Now a days the $\mathrm{PSO}^{10}$ is a swarm based optimization algorithm and it takes inspiration from a group of birds or a group of fish etc. The proposed hybrid algorithm fulfills local search by using the light intensity operation mechanism of FFA whereas the global search is accomplished by a PSO operator. Using this combination it maintains a balance between 'exploration' and 'exploitation' and enjoying the best of both the algorithms (FFA and PSO). ${ }^{12}$ The proposed method, referred to as Firefly Particle Swarm Optimization (FFPSO) has been compared with the normal PSO and FFA. The following comparative measures were used to study the (i) accuracy of the final solution, and (ii) convergence speed. Such comparison shows the superiority of the proposed algorithm. This algorithm outperformed both PSO and FFA over a few ECG benchmarks sets for the classification problem.

The ECG classification flow diagram is shown in the Fig. 1.

\section{Preprocessing}

To prove the performance of proposed technique, the usual MIT BIH arrhythmia database ${ }^{9}$ is considered. The data used in this algorithm confines to 11 recordings that consists of 5 normal, 3 LBBB and 3 RBBB for a duration of $60 \mathrm{~min}$ at $360 \mathrm{~Hz}$ sampling rate. Total number of ECG beats used for classification are 19,039. De-noising of ECG data is a preprocessing step that removes noise and makes ECG file useful for subsequent steps in the algorithm. The Sgolay FIR smoothing filter is used for removing the noise in ECG signals. The next step in the preprocessing is the $R$ peak detection, then segmentation of ECG file into beats ( $P, Q R S$ Complex), by taking $\mathrm{R}$ peaks as the reference points.

\section{Feature extraction}

In the feature extraction procedure, a fraction of signal around the $\mathrm{R}$ peak is extracted as the time-domain features since the R peaks of ECG signal are an important index for cardiac diseases. To ensure the important characteristic points of ECG like P, Q, R, S and T are included, a total of 200 sampling points before and after the R peak are collected as one ECG beat sample. The samples that are extracted from each beat contain nonuniform samples. The nonuniform samples in each beat are

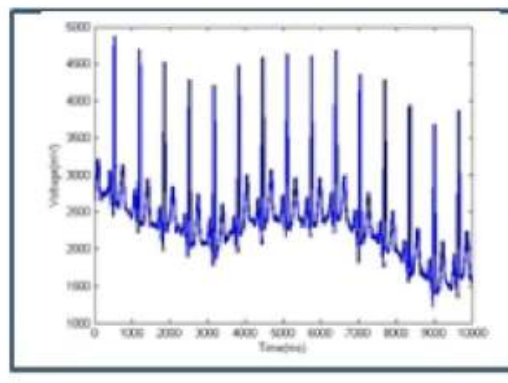

Raw ECG

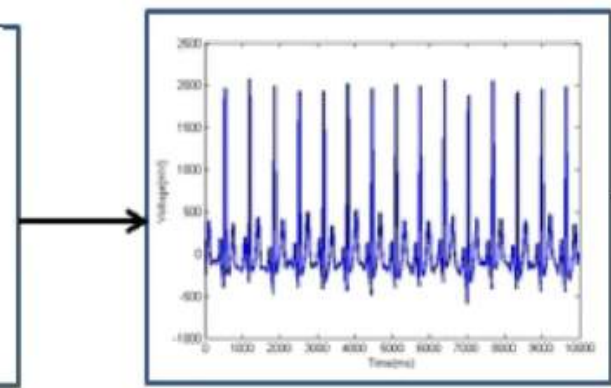

Filtered ECG

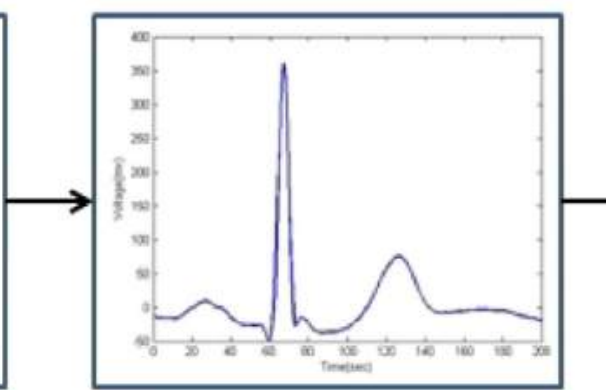

ECG Beat (features)

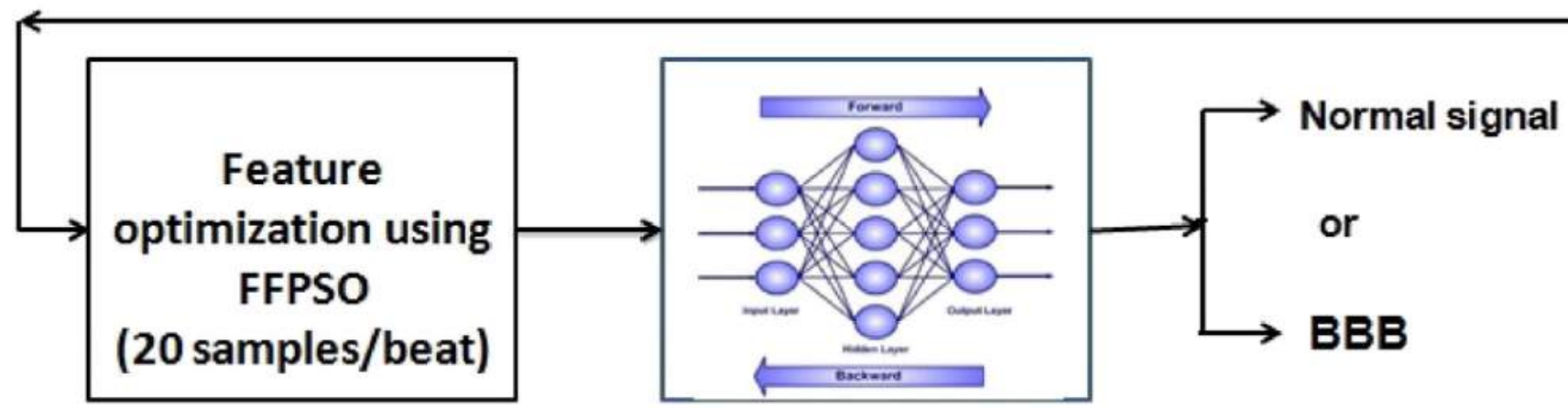

\section{Optimization of features}

\section{Classification using LMNN}

Fig. 1. ECG classification using FFPSO. 
converted into uniform samples of size 200 by using a technique called resampling. The resampled ECG beat samples/features.

\section{Feature optimization}

Particle Swarm Optimization (PSO)

$\mathrm{PSO}^{11}$ is a kind of swarm based optimization method developed by Eberhart and Kennedy inspired from the behavior of a flock of birds. Each particle in the group flies in the search domain with a velocity and it tries to attain the best velocity according to its own previous best (pbest) and its companions' best (gbest) flying experience.

The advantage of using PSO over other optimization techniques is its simplicity. And very few parameters need to be adjusted. Due to this, PSO has been widely used in a variety of applications. In an n-dimensional search space, $X_{i}=(\mathrm{x} 1, \mathrm{x} 2, \mathrm{x} 3, \ldots, \mathrm{xn})$, let the particles be initialized with positions $X_{i}$ and velocities $V_{i}$ and the fitness is calculated based on particle positional coordinates as the input values. Then the particles are moved into new positions using the equations below:

$V_{i}(i+1)=\omega \cdot V_{i}(i)+C 1 . \phi 1 .\left(\right.$ Pbest $\left.-X_{i}(i)\right)+C 2 . \phi 2 .\left(\right.$ gbest $\left.-X_{i}(i)\right)$

$X_{i}(i+1)=X_{i}(i)+V_{i}(i+1)$

\section{Firefly algorithm (FFA)}

This algorithm was designed by a mathematician X.S. Yang in the year 2007. FFA was formulated by mimicking the flashing (mating) activity of fireflies. Even though this algorithm is similar to the PSO, Artificial Bee Colony (ABC) Optimization ${ }^{14}$ and Ant Colony Optimization (ACO), ${ }^{15}$ proved to be much simpler in algorithm implementation.

Fireflies are small insects, which are capable of producing light to attract a prey (mate). They release small rhythmic light flashes. The light intensity attraction 'I' of fireflies decreases with the distance ' $r$ '. Hence, most fireflies are visible only up to several hundreds of meters. To execute this algorithm the fitness function is articulated based on the fluorescence light behavior of fireflies. For simplicity, it is imagined that light intensity attractiveness of firefly is determined by its brightness 'I' which is in turn connected with the fitness function.

\section{Attractiveness and light intensity}

At a particular position ' $r$ ', the brightness 'I' of a firefly can be chosen as I ( $r$ ), proportional to the fitness, for a maximization problem. So the I (r) varies according to the well known inverse square law.

$I(r)=\frac{I s}{r^{2}}$

Fireflies attractiveness $\beta$ is proportional to the $I(r)$ seen by surrounding fireflies can be defined as

$\beta=\beta_{0} e^{-\gamma r^{2}}$

where $\gamma$ is the light absorption coefficient.

\section{Distance}

The distance between any 2 fireflies is estimated using the distance formula.

$r_{i, j}=\sqrt{\sum_{k=1}^{d}\left(x_{i, k}-x_{j, k}\right)^{2}}$
Firefly ' $i$ ' is moved towards brighter firefly ' $\mathrm{j}$ ' and its movement is calculated by

Movement

$x_{i}=x_{i}+\beta_{0} e^{-\gamma r_{i, j}^{2}}\left(x_{i}-x_{j}\right)+\alpha \epsilon_{i}$

The first term in Eq. (6) denotes the current location of a firefly, the second term is used for determining the attractiveness $(\beta)$ of a firefly (attractive firefly), towards the attractive neighboring fireflies and the third term indicates the random walk of a firefly (random part).

$x i=x i+\alpha($ rand $-1 / 2)$

when firefly 'i' lacks the brighter firefly ' $\mathrm{j}$ ' then it will go for a random walk as in Eq. (7), in search of the best candidate, where the coefficient $\alpha$ is a randomization variable, and 'rand' is a random number consistently spread over the space $(0,1)$.

The pseudo code for firefly algorithm is given below

Pseudo code: firefly algorithm

1. Generate the initial population randomly.

2. Calculate the fitness of initial population based on light intensity of fireflies.

3. While ( $\mathrm{t}<$ termination criteria is satisfied)

4. For $\mathrm{i}=1: \mathrm{p}$ ( $\mathrm{p}$ fireflies)

5. For $\mathrm{j}=1: \mathrm{p}$

6. Calculate light intensity (I) using Eq. (3).

7. Distance between two fireflies is calculated using Eq. (5).

8. If $(\mathrm{I}(\mathrm{i})<\mathrm{I}(\mathrm{j}))$

9. Firefly $i$ is moved towards firefly j using Eq. (6).

10. Determine new solutions.

11. Else

12. Firefly i is moved randomly towards j using Eq. (7).

13. End If

14. End for $\mathrm{j}$.

15. End for $i$

16. End while

17. Sort the fireflies according to light intensity values of the new solution.

Proposed approach: hybrid FF and PSO (FFPSO) algorithm

In this section, a part of PSO is used in the FFA to increase convergence and also to enhance its capability for not falling into the local minimum. The FFPSO has exactly the same steps as the FFA with the exception that the position vector of FFA is modified as follows: In the FFPSO, the distance between xi and pbest $_{i}$, is the Cartesian distance

$r_{p x}=\sqrt{\sum_{k=1}^{d}}\left(\text { pbest }_{i, j}-x_{i, j}\right)^{2}$

The distance between $\mathrm{xi}$ and gbest $_{i}$, is the Cartesian distance

$r_{g x}=\sqrt{\sum_{k=1}^{d}}\left(\text { gbest }_{i, j}-x_{i, j}\right)^{2}$

The position vectors $x i$ of the FFPSO is randomly mutated by using Eq. (8)

$x_{i}(t+1)=w x_{i}(t)+c_{1} e^{-r_{p x}^{2}}\left(\right.$ pbest $\left._{i}-x_{i}(t)\right)$
$+c_{2} e^{-r_{g x}^{2}}\left(\right.$ gbest $\left._{i}-x_{i}(t)\right)+\alpha(\gamma-1 / 2)$ 


\section{Pseudo code: FFPSO}

1. Generate the initial population randomly.

2. Initialize pbest and gbest.

3. Calculate the fitness of initial population based on light intensity of fireflies.

4. While (stopping criteria is satisfied)

5. For $\mathrm{i}=1: \mathrm{p}$ ( $\mathrm{p}$ fireflies)

6. For $\mathrm{j}=1: \mathrm{p}$

7. Light intensity I is determined using Eq. (3).

8. Distance between pbest-xi and gbest-xi is calculated using Eqs. (8) and (9).

9. If $(\mathrm{I}(\mathrm{i})<\mathrm{I}(\mathrm{j}))$

10. Firefly $i$ is moved towards firefly j using Eq. (10)

11. Else

12. Firefly i is moved randomly towards firefly j using Eq. (7).

13. End If

14. Calculate the new solutions and update the light intensity value

15. Update pbest and gbest.

16. End for $\mathrm{j}$

17. End for i

18. End while

19. Sort the fireflies in descending order based on their light intensity

In the suggested approach, the light intensity attraction step of each particle gets mutated by a PSO operator. At this step, each particle is randomly attracted towards the gbest position in the entire population. Local search in different regions is carried by the modified attractiveness step of the FFPSO algorithm. The main objective of FFPSO feature selection stage is to reduce the features of the problem before the supervised neural network classification. Among all the wrapper algorithms used, FFPSO, which solves optimization problems using the methods of flashing behavior of fireflies, has emerged as a promising one.

\section{Classification of ECG with firefly features}

The extracted features from FFPSO algorithm (20 features) are classified using different types of classification techniques such as K-Nearest Neighbor (KNN), Support Vector Machine (SVM), LM Neural Network classifiers.

\section{Levenberg-Marquardt Neural Network (LM NN)}

In this work for the detection of BBB, back propagation LevenbergMarquardt Neural Network (LMNN) ${ }^{19}$ was used. This NN provides rapid execution of the network to be trained, which is the main advantage in the neural signal processing applications, ${ }^{8}$ citeKP. The NN was designed to work well if it was built with 20 input neurons, 10 neurons in the hidden layer and 3 neurons in the output layer. The performance of this algorithm was compared with Scalar Conjugate Gradient (SCG) NN. The LMNN algorithm is a robust and a very simple method for approximating a function. SCG NN method provides conjugate directions of search instead of performing a linear search. The network is trained with 11,039 ECG beats, and tested with 8000 ECG beats. The total number of iterations is set to 1000 and mean square error less than 0.001 . The main advantage of this algorithm is that the time required to train the network is less.

\section{Results}

ECG features before optimization $=\left[\begin{array}{llll}1 & 2 & 3 & \text {.........200]; }\end{array}\right]$

The optimized features are $=[41,14,198,17,189,139,22,81,177$, $1,171,82,134,40,49,38,80,86,129,138]$;

These reduced features are given as input for the Neural Network so that its convergence speed and final accuracy can be increased. The ECG beats after segmentation are re-sampled to 200 samples/beat. Instead of using morphological feature extraction techniques, in this paper FFPSO is used as the feature extraction technique. Using FFPSO ECG beat features are optimized to 20 features. The FFPSO gives optimized features (best features) for the classification. The performance of FFPSO is compared with classical FFA and PSO techniques. The FFA, PSO and FFPSO features are classified using SVM, KNN, SCG NN and LM NN as in Table 1.

- Count of Normal beats used for classification-9193.

- Count of RBBB beats user for classification-3778.

- Count of LBBB beats user for classification-6068.

- Total number of beats used for classification-19,039.

- Count of correctly classified beats-18,800.

- Total misclassified beats-239.

For measuring accuracy two parameters sensitivity and specificity are calculated using the following equations.

$$
\begin{aligned}
& \text { Specificity }=\frac{\text { True_Negative }}{\text { True_Negative }+ \text { False_Positive }} X 100 \\
& \text { Sensitivity }=\frac{\text { True_Positive }}{\text { True_Positive }+ \text { False_Negative }} X 100 \\
& \text { Accuracy }=\frac{T P+T N}{T P+T N+F P+F N} X 100
\end{aligned}
$$

- TP (True_Positive $)=$ count of all the correctly classified normal beats.

- TN (True_Negative) = count of all beats the correctly classified abnormal beats.

- FP (False_Positive $)=$ count of normal beats which are classified as abnormal.

- FN (False_Negative) = count of abnormal beats which are classified as normal.

In the training mode we applied multilayer NN and checked the network performance and decided if any changes were required to the training process or the data set or the network architecture.

\section{Discussion}

The proposed FFPSO is compared against other three BBB detection algorithms such as Wavelet Transform (WT), Continuous wavelet Transform (CWT), Wavelet transform and Probabilistic Neural Network (PNN) in terms of related features selected from the original database and classification accuracy obtained from different classifiers using Matlab software.

The work in, ${ }^{18}$ explores an experimental study of using WT for extracting relevant features and KNN based classifier for the detection of BBB. The work presented in, ${ }^{17}$ uses morphological features for classification using SVM. The work proposed in, ${ }^{16}$ uses Arrhythmia dataset

Table 1

Classification with LM NN classifier.

\begin{tabular}{llll}
\hline Classifier & Sensi & Speci & Accuracy \\
\hline FFA + SVM & $76.2 \%$ & $75.47 \%$ & $72.13 \%$ \\
PSO + SVM & $71.0 \%$ & $73.13 \%$ & $70.12 \%$ \\
FFPSO + SVM & $95.5 \%$ & $96.9 \%$ & $96.74 \%$ \\
FFA + SCG NN & $88.2 \%$ & $87.2 \%$ & $87.9 \%$ \\
PSO + SCG NN & $86.1 \%$ & $85.3 \%$ & $86.0 \%$ \\
FFPSO + SCG NN & $97.42 \%$ & $92.28 \%$ & $97.13 \%$ \\
FFA + KNN & $53.5 \%$ & $52.2 \%$ & $53.22 \%$ \\
PSO + KNN & $52.5 \%$ & $53.2 \%$ & $65.1 \%$ \\
FFPSO + KNN & $92.35 \%$ & $93.9 \%$ & $92.17 \%$ \\
FFA + LM NN & $93.34 .2 \%$ & $92.2 \%$ & $93.9 \%$ \\
PSO + LM NN & $91.2 \%$ & $89.2 \%$ & $80.9 \%$ \\
FFPSO + LM NN & $99.97 \%$ & $98.7 \%$ & $99.1 \%$ \\
\hline
\end{tabular}


Table 2

Comparative study for detection of BBB.

\begin{tabular}{|c|c|c|}
\hline Studies & Approach & Accuracy \\
\hline R Ceylan et al. (2011) ${ }^{17}$ & Wavelet Transform (WT) & $98.1 \%$ \\
\hline Kutlu et al. $(2008)^{18}$ & Continuous Wavelet Transform (CWT) & $97.3 \%$ \\
\hline Yu et al. $(2007)^{16}$ & WT and Probabilistic Neural Network & $98.39 \%$ \\
\hline Proposed approach & FFPSO and Neural Network & $99.1 \%$ \\
\hline
\end{tabular}

taken from MIT/BIH repository and 20 morphological and wavelet features are extracted then PNN is used for supervised learning and classification. From the experiments, it is concluded that the proposed FFPSO with LMNN classifier outperformed other three algorithms with selection of minimal number of relevant features. This increases the classification accuracy as shown in Table 2. The FFPSO employed to intelligently select the most relevant features that could increase the classification accuracy while ignoring noisy and redundant features.

\section{Conclusion}

It is evident from the results that hybrid FFPSO approach out performs the other two optimization methods in terms of accuracy and convergence rates. In the present study, we developed a simple computational model for the detection of ECG BBB using the FFPSO algorithm. The FFPSO algorithm was compared with the FFA and PSO . In our study the following were observed: 1) accuracy 2) convergence speed. The FFPSO method was shown to provide better results than original FFA and PSO for all the tested data.

\section{References}

1. Mishra Anurag, et al. Optimized gray-scale image watermarking using DWT - SVD and firefly algorithm. Expert Syst Appl 2014;41(17):7858-7867.

2. Sahu Rabindra Kumar, Panda Sidhartha, Padhan Saroj, et al. A hybrid firefly algorithm and pattern search technique for automatic generation control of multi area power systems. Int J Electr Power Energy Syst 2015;64:9-23.
3. Nasir Ahmad NK, Tokhi MO, Ghani N, et al. Novel adaptive bacteria foraging algorithms for global optimization. Applied Computational Intelligence and Soft Computing; 2014.

4. Passino Kevin M. Biomimicry of bacterial foraging for distributed optimization and control. Control Syst 2002;22(3):52-67.

5. Liu Y, Passino KM, et al. Biomimicry of social foraging bacteria for distributed optimization models, principles, and emergent behaviors. J Optim Theory Appl 2002;115(3): 603-628.

6. Kennedy J, Eberhart R, et al. Particle swarm optimization. Proc. IEEE Int. Conf. Neural Netw; 1995. p. 1942-1948.

7. Back T. Evolutionary algorithms in theory and practice: evolution strategies, evol. programming. Proc. Genetic Algorithms. London, U.K.: Oxford Univ. Press; 1996

8. Sapna S, Tamilarasi A, Kumar MP, et al. Backpropagation learning algorithm based on levenberg marquardt algorithm. CS and IT-CSCP; 2012;393-398.

9. Goldberger AL, Amaral LA, Glass L, Hausdorff JM, Ivanov PC, Mark RG, Stanley HE et al. Physiobank, physiotoolkit, and physionet components of a new research resource for complex physiologic signals. Circulation 2000;101(23):e215-e220.

10. Eberhart RC, Kennedy J, et al. A new optimizer using particle swarm theory. Proc. ISMMH S; 1995. p. 39-43.

11. Melgani F, Bazi Y, et al. Classification of electrocardiogram signals with support vector machines and particle swarm optimization. IEEE Trans Inf Technol Biomed 2008;12(5).

12. Abd-Elazim SM, Ali ES, et al. A hybrid particle swarm optimization and bacterial foraging for optimal power system stabilizers design. Int J Electr Power Energy Syst 2013;46:334-341.

13. Horng Ming-Huwi, et al. Firefly meta-heuristic algorithm for training the radial basis function network for data classification and disease diagnosis. INTECH Open Access Publisher; 2012.

14. Pal SK, Rai CS, Singh AP, et al. Comparative study of firefly algorithm and particle swarm optimization for noisy non-linear optimization problems. Int J Intell Syst Appl 2002;4(10):50.

15. Dorigo Marco, Stutzle Thomas. The ant colony optimization metaheuristic: algorithms, applications, and advances. Handbook of metaheuristics. US: Springer; 2003;250-285.

16. Yu Wei, et al. Application of multi-objective genetic algorithm to optimize energy efficiency and thermal comfort in building design. Energy Build 2015;88:135-143.

17. Ceylan Rahime, ozbay Yksel, et al. Wavelet neural network for classification of bundle branch blocks. Proc World Congr Eng 2011;4(2).

18. Kutlu Yakup, Kuntalp Damla, Kuntalp Mehmet, et al. Arrhythmia classification using higher order statistics. Signal Processing, Communication and Applications Conference; 2008.

19. Kora Padmavathi, Sri Rama Krishna K. Improved Bat algorithm for the detection of myocardial infarction. SpringerPlus 2015;4(1):666. 